\title{
On the Hardness of the Determinant: Sum of Regular Set-Multilinear Circuits
}

\author{
S. Raja* Sumukha Bharadwaj G $\mathrm{V}^{\dagger}$
}

\begin{abstract}
In this paper, we study the computational complexity of the commutative determinant polynomial computed by a class of setmultilinear circuits which we call regular set-multilinear circuits. Regular set-multilinear circuits are commutative circuits with a restriction on the order in which they can compute polynomials. A regular circuit can be seen as the commutative analogue of the ordered circuit defined by Hrubes, Wigderson and Yehudayoff $[\underline{5}$. We show that if the commutative determinant polynomial has small representation in the sum of constantly many regular set-multilinear circuits, then the commutative permanent polynomial also has a small arithmetic circuit.
\end{abstract}

\section{Introduction}

Arithmetic circuit complexity studies the complexity of computing polynomials using arithmetic operations. Arithmetic circuits are a natural computational model for computing and describing polynomials. Arithmetic circuit is a directed acyclic graph with internal nodes labeled by + or $\times$, and leaves labeled by either variables or elements from a underlying field $\mathbb{F}$. The complexity measures associated with arithmetic circuits are size, which measures number of gates in the circuit, and depth, which measures length of the longest path from a leaf to the output gate in the circuit. Two important examples of polynomial family are the determinant and the permanent polynomials. The determinant polynomial is ubiquitous in linear algebra, and it can be computed by polynomial-sized arithmetic circuits (see e.g., [3]). On the other hand, the permanent of $0 / 1$ matrices is \#P-complete [10], where \#P corresponds to the counting class in the world of Boolean

\footnotetext{
*IIT Tirupati, India, email: raja@iittp.ac.in

${ }^{\dagger}$ IIT Tirupati, India, email: cs21d003@iittp.ac.in
} 
complexity classes. Thus, it is believed that, over fields of characteristic different from 2 , the permanent $P E R M=\left(P E R M_{n}\right)$ polynomial family cannot be computed by any polynomial-sized circuit family. A central open problem of the field is proving super-polynomial size lower bounds for arithmetic circuits that compute the permanent polynomial $P E R M_{n}$. Motivated by this problem, Valiant, in his seminal work [9], defined the arithmetic analogues of $\mathrm{P}$ and NP: denoted by VP and VNP. Informally, VP consists of multivariate (commutative) polynomials that have polynomial size circuits. Valiant showed that $P E R M$ is VNP-complete w.r.t. projection reductions. Thus, $V P \neq V N P$ iff $P E R M_{n}$ requires arithmetic circuits of size superpolynomial in $n$.

Set-multilinear circuits are introduced in the work of [7]. Let $\mathbb{F}$ be a field and $X=X_{1} \sqcup X_{2} \sqcup \cdots \sqcup X_{d}$ be a partition of the variable set $X$. A set-multilinear polynomial $f \in \mathbb{F}[X]$ w.r.t. this partition is a homogeneous degree $d$ multilinear polynomial such that every nonzero monomial of $f$ has exactly one variable from $X_{i}$, for all $1 \leq i \leq d$. Some of the wellknown polynomial families like the permanent $P E R M_{n}$ and the determinant $D E T_{n}$, are set-multilinear. The variable set is $X=\left\{x_{i j}\right\}_{1 \leq i, j \leq n}$ and the partition can be taken as the row-wise partition of the variable set. I.e. $X_{i}=\left\{x_{i j} \mid 1 \leq j \leq n\right\}$ for $1 \leq i \leq n$. In this work, we study the set-multilinear circuit complexity of the determinant polynomial $D E T_{n}$. A set-multilinear arithmetic circuit $C$ computing $f$ w.r.t. the above partition of $X$, is a directed acyclic graph such that each in-degree 0 node of the graph is labeled with an element from $X \cup \mathbb{F}$. Each internal node $v$ of $C$ is of in-degree 2 , and is either a + gate or $\times$ gate. With each gate $v$ we can associate a subset of indices $I_{v} \subseteq[d]$ and the polynomial $f_{v}$ computed by the circuit at $v$ is set-multilinear over the variable partition $\bigsqcup_{i \in I_{v}} X_{i}$. If $v$ is a + gate then for each input $u$ of $v, I_{u}=I_{v}$. If $v$ is a $\times$ gate with inputs $v_{1}$ and $v_{2}$ then $I_{v}=I_{v_{1}} \sqcup I_{v_{2}}$. Clearly, in a set-multilinear circuit every gate computes a set-multilinear polynomial (in a syntactic sense). The output gate of $C$ computes the polynomial $f$, which is set-multilinear over the variable partition $\bigsqcup_{i \in[d]} X_{i}$. The size of $C$ is the number of gates in it and its depth is the length of the longest path from an input gate to the output gate of $C$. Additionally, a set-multilinear circuit $C$ is called a set-multilinear formula if out-degree of every gate is bounded by 1 .

Set-multilinear arithmetic circuits are a natural model for computing set-multilinear polynomials. It can be seen that each set-multilinear polynomial can be computed by a set-multilinear arithmetic circuit. For setmultilinear formulas, super-polynomial size lower bounds are known [8]. Super-polynomial lower bounds for a class of set-multilinear ABPs comput- 
ing the determinant $D E T_{n}$ is shown in [1]. It is known that proving superpolynomial lower bound result for general set-multilinear circuits computing the permanent polynomial $P E R M_{n}$ would imply that $P E R M_{n}$ requires super-polynomial size non-commutative arithmetic circuits, and this is an open problem for over three decades. Non-commutative circuits are a restriction on the computational power of circuits. Though non-commutative circuits compute non-commutative polynomials, one can study what is the power of commutativity in computing the $D E T_{n}$ polynomial. Noncommutative arithmetic circuit models are well studied, see e.g., [6, 2, 5]. In [2], it was shown that computing the non-commutative determinant polynomial is as hard as computing the commutative permanent polynomial.

\subsection{Our Results}

To explain our results, we first define the computational model that we study. Let $S_{n}$ denote the set of all permutations over the set $\{1,2 \ldots, n\}$.

Definition 1 (Regular Set-Multilinear Circuits). Let $X=X_{1} \sqcup X_{2} \sqcup \cdots \sqcup$ $X_{d}$ be a partition of the variable set $X$. Let $\sigma \in S_{d}$. A set-multilinear circuit $C$ that computes a set-multilinear polynomial $f \in F[X]$ w.r.t the above partition is called regular set-multilinear circuit w.r.t $\sigma \in S_{d}$, if every gate $v$ in $C$ is associated with an interval $I_{v}$ w.r.t $\sigma \in S_{d}$. In other words, $\sigma \in S_{d}$ defines an ordering $(\sigma(1), \sigma(2), \cdots, \sigma(d))$ and every gate $v$ in $C$ is associated with an interval $I_{v}$ w.r.t $\sigma$-ordering $(\sigma(1), \sigma(2), \cdots, \sigma(d))$.

Let $C$ be a regular set-multilinear circuit w.r.t $\sigma$ computing a commutative polynomial $f$ of degree $d$. Let $v$ be a gate in $C$ computing the polynomial $f_{v}$ of degree $k$. By definition, $f_{v}$ is a set-multilinear polynomial w.r.t $I_{v}=[\sigma(i), \sigma(i+1), \cdots, \sigma(i+k)]$, where $i<=d-k$. Let $\operatorname{order}\left(f_{v}\right)=I_{v}=(\sigma(i), \sigma(i+1), \cdots, \sigma(i+k))$.

Since for each gate $v$ in $C, I_{v}$ can be viewed as an interval w.r.t $\sigma \in S_{d}$, the two children $u$ and $w$ of $v$ can be designated as left and right child. In particular, for each product gate $v$ with children $u$ and $w$ such that $I_{v}=I_{u} \sqcup I_{w}$, we refer to $u$ as the left child of $v$, and $w$ as the right child of $v$.

We make the following observations about regular set-multilinear circuits:

- If $v$ is an input gate (leaf node) labeled by a field constant, then $\operatorname{order}\left(f_{v}\right)=()$, where () is the empty sequence. If $v$ is an input gate labeled by a variable $x_{i, j}$, then $\operatorname{order}\left(f_{v}\right)=(i)$. 
- If $v$ is an product gate, then $\operatorname{order}\left(f_{v}\right)=\operatorname{order}\left(f_{u}\right) \sqcup \operatorname{order}\left(f_{w}\right)$, where the interval $\operatorname{order}\left(f_{v}\right)$ is obtained by appending $\operatorname{order}\left(f_{u}\right)$ with $\operatorname{order}\left(f_{w}\right)$.

- If $v$ is a sum gate, then $\operatorname{order}\left(f_{v}\right)=\operatorname{order}\left(f_{u}\right)=\operatorname{order}\left(f_{w}\right)$.

One can define several versions of non-commutative $D E T_{n}$ polynomial. Non-commutative circuits computing the $D E T_{n}$ polynomial, where the first index of the variables in each monomial is in increasing order, can be seen as regular set-multilinear w.r.t the identity permutation. In [2], it was shown that computing the non-commutative determinant polynomial is as hard as computing the commutative permanent polynomial. A natural next step is to find the set-multilinear circuit complexity of the commutative determinant polynomial.

We study the computational complexity of the commutative determinant polynomial $D E T_{n}$ computed by a sum of regular set-multilinear circuits. We show that if the determinant polynomial $D E T_{n}$ is computed by a circuit $C$ of size $s$, where $C$ is a sum of constantly-many regular setmultilinear circuits, then we can modify $C$ to compute the permanent polynomial $P E R M_{n^{\epsilon}}$, where $\epsilon>0$, such that the new circuit size is polynomially related to the size of $C$. We remark that in our result, there is no restriction on the number of different parse tree types/shapes (see e.g., [1]) allowed in each regular circuits.

One can view this as a generalization of the result shown in [2] to a class of set-multilinear circuits computing the determinant polynomial $D E T_{n}$. We obtain our result by carefully combining Erdös-Szekeres theorem [4] and some properties that we prove about regular set-multilinear circuits and the result of [2].

\section{Preliminaries}

\subsection{Determinant and Permanent}

Definition 2. (Commutative Determinant and Permanent) Given the set of variables $X=\left\{x_{i, j} \mid 1 \leq i, j \leq n\right\}$, the $n \times n$ commutative determinant and the $n \times n$ commutative permanent over $X$, denoted by $D E T_{n}(X)$ and $P E R M_{n}(X)$ respectively, are $n^{2}$-variate polynomials of degree $n$ given by:

$$
\operatorname{DET}_{n}(X)=\sum_{\sigma \in S_{n}} \operatorname{sgn}(\sigma) \prod_{i=1}^{n} x_{i, \sigma(i)}
$$




$$
\operatorname{PERM}_{n}(X)=\sum_{\sigma \in S_{n}} \prod_{i=1}^{n} x_{i, \sigma(i)},
$$

Non-commutative determinant can be defined in various ways depending on the order in which variables are multiplied. One natural type of noncommutative determinant, called the Cayley determinant $C D E T_{n}$, is one where the order of multiplication is the identity permutation w.r.t first index of the variable.

\subsection{Erdös-Szekeres Theorem}

Theorem 1 (Erdös-Szekeres Theorem, 4]). Let $n$ be a positive integer. Let $S$ be a sequence of distinct integers of length at least $n^{2}+1$. Then, there exists a monotonically increasing subsequence of $S$ of length $n+1$, or a monotonically decreasing subsequence of $S$ of length $n+1$.

Let $A, B$ be two $n \times n$ matrices. The following are known facts about the determinant and permutations.

Fact 1: $\operatorname{det}(A \times B)=\operatorname{det}(A) \times \operatorname{det}(B)$.

Fact 2: The determinant of a permutation matrix is either +1 or -1 .

Fact 3: Let $\tau, \sigma \in S_{n}$. Then $\operatorname{sign}(\tau \circ \sigma)=\operatorname{sign}(\tau) \times \operatorname{sign}(\sigma)$.

For $n \in \mathbb{N}$, let $[n]=\{1,2, \cdots, n\}$.

\section{Hardness of the Determinant: Sum of Two Reg- ular Set-Multilinear Circuits}

In this section, we show that if the determinant polynomial is computed by a sum of two regular set-multilinear circuits then the permanent polynomial can also be represented as a regular set-multilinear circuit. This result involves all the techniques which will be used in the main result and it is easy to explain in this sum of two regular circuits model. In the next section, we will prove the result for sum of constantly many regular set-multilinear circuits. We note that all our polynomials are commutative. For the purpose of readability, we sometimes ignore the floor operation.

Let $X=\left\{x_{i, j} \mid 1 \leq i, j \leq n\right\}$ be the set of variables. Let $X_{i}=\left\{x_{i j} \mid 1 \leq\right.$ $j \leq n\}$ for $1 \leq i \leq n$. Our aim is to show that if $C=C_{1}^{\sigma 1}+C_{2}^{\sigma 2}$ computing the determinant polynomial $D E T_{n}(X) \in \mathbb{F}[X]$, where the circuits $C_{1}^{\sigma 1}, C_{2}^{\sigma 2}$ are regular set-multilinear circuits w.r.t $\sigma 1, \sigma 2 \in S_{n}$ respectively, then there is an efficient transformation that converts the given circuit $C$ to another 
circuit $C^{\prime}$ computing the permanent polynomial of degree $\sqrt{n} / 2$. Given $C=C_{1}^{\sigma 1}+C_{2}^{\sigma 2}$ computing $D E T_{n}(X)$, if $\sigma 1=\sigma 2$ then we can directly adapt the result of [2] and get a circuit $C^{\prime}$ computing the permanent polynomial of degree $n / 2$. If $n$ is not even then we can substitute variables in the set $X_{n}$ suitably from $\{0,1\}$ such that $C$ computes $D E T_{n-1}(X)$ before using the result of [2].

The case of $\sigma 1 \neq \sigma 2$ needs more work that we explain now. The idea is to use the well known Erdös-Szekeres Theorem [4] that guarantees that any sequence of $n$ distinct integers contains a subsequence of length at least $\sqrt{n}$ that is either monotonically increasing or decreasing. By viewing $\sigma=(\sigma(1), \sigma(2), \cdots, \sigma(n))$ as a sequence of integers, we apply the above result to permutations $\sigma 1, \sigma 2 \in S_{n}$. We first apply it to $\sigma 1=(\sigma 1(1), \sigma 1(2), \ldots, \sigma 1(n))$ and let $A=\left\{i_{1}, i_{2}, \cdots, i_{\sqrt{n}}\right\}$ be the set of indices that appear in this monotone subsequence. If the subsequence is monotonically increasing then we do substitutions in $D E T_{n}(X)$ so that it computes the determinant polynomial of $\sqrt{n} \times \sqrt{n}$ matrix whose rows and columns are labeled by the elements of set $A$. This is done by making suitable substitutions to the variables in $X$ from $X \cup\{0,1\}$ in the given circuit $C$. After this we get a circuit $C^{\prime}$ from $C$ that computes $D E T_{\sqrt{n}}\left(X^{\prime}\right)$ where $X^{\prime}=\bigsqcup_{i \in A} X_{i}$.

We note that $C^{\prime}=C_{1}^{\sigma 1^{\prime}}+C_{2}^{\sigma 2^{\prime}}$ where $\sigma 1^{\prime}, \sigma 2^{\prime} \in S_{\sqrt{n}}$ and $\sigma 1^{\prime}=$ $\left(\sigma 1^{\prime}(1), \sigma 1^{\prime}(2), \cdots, \sigma 1^{\prime}(\sqrt{n})\right)$ is in increasing order. If $\sigma 1^{\prime}=\sigma 2^{\prime}$, then we can use [2] and get the permanent of degree $\sqrt{n} / 2$. Otherwise, we apply Erdös-Szekeres Theorem to permutations $\sigma 1^{\prime}, \sigma 2^{\prime}$. In particular, this will give us a monotone subsequence in $\sigma 2^{\prime}=\left(\sigma 2^{\prime}(1), \sigma 2^{\prime}(2), \cdots, \sigma 2^{\prime}(\sqrt{n})\right)$ with length at least $n^{1 / 4}$. If this sequence is increasing, then the same subsequence is also increasing in $\sigma 1^{\prime}$ as we already noted that it is in increasing order. Let $A_{1}=\left\{j_{1}, j_{2}, \cdots, j_{n^{1 / 4}}\right\}$ be the set of indices that appear in this monotone subsequence. Now we project, as before so that it computes the determinant polynomial of a $n^{1 / 4} \times n^{1 / 4}$ matrix whose rows and columns are labeled by the elements in set $A_{1}$. After substituting from $X^{\prime} \cup\{0,1\}$ for each variable in the given circuit $C^{\prime}$, we get a regular circuit $C^{\prime \prime}$ that computes $D E T_{n^{1 / 4}}\left(X^{\prime \prime}\right)$, where $X^{\prime \prime}=\bigsqcup_{i \in A_{1}} X_{i}$.

The important thing to note here is that in the new circuit $C^{\prime \prime}=$ $C_{1}^{\sigma 1^{\prime \prime}}+C_{2}^{\sigma 2^{\prime \prime}}$, where $\sigma 1^{\prime \prime}, \sigma 2^{\prime \prime} \in S_{n^{1 / 4}}$, both $\sigma 1^{\prime \prime}$ and $\sigma 2^{\prime \prime}$ are the same, i.e., $\sigma 1^{\prime \prime}=\sigma 2^{\prime \prime}$. We can rename the variable sets in $X^{\prime \prime}=\bigsqcup_{i \in A_{1}} X_{i}$ to $X_{1}, X_{2}, \cdots, X_{n^{1 / 4}}$. For example, if $i_{1} \in A_{1}$ is the lowest index then we can rename $X_{i_{1}}$ to $X_{1}$, and for all $j$, rename $X_{i_{1}, j}$ to $X_{1, j}$. Similarly, the $k$-th lowest index is modified. After these modifications, we can assume that 
$\hat{X}=\bigsqcup_{i \in\left[n^{1 / 4}\right]} X_{i}$.

As we noted before, any non-commutative circuit computing $D E T_{n}$, where the first index of the variables in each monomial is in increasing order, can be seen as regular set-multilinear w.r.t identity permutation. Now we can apply the following theorem (Theorem 10 from [2]) to get our result.

Theorem 2. (Theorem 10, [2]) For any $n \in \mathbb{N}$, if there is a noncommutative circuit $C$ of size $s$ computing the Cayley determinant $D_{E T} T_{2 n}(X)$ then there is a circuit $C^{\prime}$ of size polynomial in $s$ and $n$ that computes the Cayley permanent PERM $M_{n}(Y)$.

If $n^{\prime}=\left\lfloor n^{1 / 4}\right\rfloor$ is not an even number then we ignore the $X_{n^{\prime}}$ variable set in $\hat{X}$ by following substitutions: $X_{n^{\prime}, n^{\prime}}=1$ and for all $j \in\left[n^{\prime}-1\right], X_{n^{\prime}, j}=0$ and $X_{j, n^{\prime}}=0$. After this substitutions, we have a circuit that computes the determinant $D E T_{n^{\prime}-1}$ polynomial. Now applying the above theorem we get a circuit $\widehat{C}$ that computes the permanent polynomial of degree $\frac{n^{\prime}-1}{2}$.

We now explain how to handle if Erdös-Szekeres Theorem guarantees only monotonically decreasing sequence. For that we define the reverse of a regular set-multilinear circuit $C$ w.r.t $\sigma \in S_{n}$ computing a polynomial $f$. This results in a regular set-multilinear circuit $C^{\text {rev }}$ w.r.t $\sigma^{\text {rev }} \in S_{n}$, where $\sigma^{r e v}=(\sigma(n), \sigma(n-1), \ldots, \sigma(1))$, computing the same commutative polynomial $f$ as circuit $C$. We note that if $\sigma$ has monotonically decreasing subsequence of length $k$ then $\sigma^{r e v}$ has a monotonically increasing subsequence of same length $k$. We obtain $C^{\text {rev }}$ by interchanging the left and right children of product gates in $C$. This is proved in the following lemma.

Lemma 1 (Reversal Lemma). Let $X=\left\{x_{i, j} \mid 1 \leq i, j \leq n\right\}$ be a set of variables and $X=X_{1} \sqcup X_{2} \sqcup \ldots \sqcup X_{n}$ be a partition of $X$, where for all $1 \leq i \leq n, X_{i}=\left\{x_{i, 1}, x_{i, 2}, \ldots, x_{i, n}\right\}$. Let $C$ be a regular set-multilinear circuit w.r.t a permutation $\sigma \in S_{n}$ computing the polynomial $f \in F[X]$. Then, there exists a regular set-multilinear circuit $C^{\text {rev }}$ w.r.t $\sigma^{\text {rev }} \in S_{n}$ where $\sigma^{r e v}=(\sigma(n), \sigma(n-1), \ldots, \sigma(1))$ computing the same commutative polynomial $f$ as circuit $C$. Moreover, the size of $C^{\text {rev }}$ is same as that of $C$.

Proof. First, we describe the construction of the circuit $C^{r e v}$, and then prove its correctness. Let $v$ be a gate in $C$. As $C$ is a regular set-multilinear circuit w.r.t $\sigma \in S_{n}$, we have an interval $I_{v}$ w.r.t the permutation $\sigma$ associated with the gate $v$.

Construction of $C^{r e v}$ : Starting with the product gates at the bottom of $C$ and gradually moving up level-by-level, swap the left and right children of each product gate. 
Correctness: We show by induction on depth $d$ of $C$ that both circuits $C$ and $C^{r e v}$ compute the same polynomial $f \in F[X]$ and $C^{r e v}$ is a regular setmultilinear circuit w.r.t $\sigma^{\text {rev }} \in S_{n}$, where $\sigma^{\text {rev }}=(\sigma(n), \sigma(n-1), \ldots, \sigma(1))$. Let $f_{v}$ and $f_{v}^{r e v}$ denote the polynomials computed at any node $v$ in $C$ and $C^{r e v}$, respectively. Let $\operatorname{order}\left(f_{v}\right)=I_{v}$. We will show that $f_{v}$ and $f_{v}^{r e v}$ are the same polynomial and the only difference is in their orders. That is, $\operatorname{order}\left(f_{v}^{r e v}\right)=\operatorname{rev}\left(\operatorname{order}\left(f_{v}\right)\right)$, where $\operatorname{rev}\left(\operatorname{order}\left(f_{v}\right)\right)$ is $\operatorname{order}\left(f_{v}\right)$ written in reverse (i.e., the interval $I_{v}$ is reversed).

The proof is by induction on the depth $d$ of the circuit $C^{r e v}$. Let $f^{r e v}$ denote the polynomial computed by $C^{r e v}$.

Base Case: The base case is any node at depth 0, i.e., a leaf node. Consider any leaf node $l$. Then $f_{l}$, the polynomial computed at $l$, is either a variable or a field constant in $F$. If $f_{l}$ is a field constant, then $\operatorname{order}\left(f_{l}\right)=()$. Therefore, $\operatorname{order}\left(f_{l}^{r e v}\right)=()$. If $f_{l}$ is a variable $x_{i, j}, 1 \leq i, j \leq n$, then $\operatorname{order}\left(f_{l}\right)=(i)$. Therefore, the $\operatorname{order}\left(f_{l}^{r e v}\right)=(i)$. In both cases, $f_{l}^{r e v}=f_{l}$ and $\operatorname{order}\left(f_{l}^{r e v}\right)=\operatorname{rev}\left(\operatorname{order}\left(f_{l}\right)\right)$.

Induction Hypothesis: Assume for any node $u$ at depth $d^{\prime}, 1 \leq d^{\prime} \leq$ $d-1$, that $f_{u}^{r e v}=f_{u}$ and $\operatorname{order}\left(f_{u}^{r e v}\right)=\operatorname{rev}\left(\operatorname{order}\left(f_{u}\right)\right)$.

Induction Step: Consider any node $v$ at depth $d^{\prime}+1$, with $v_{L}$ and $v_{R}$ as its left and right children, respectively. By induction hypothesis, $f_{v_{L}}^{r e v}=f_{v_{L}}$ and $\operatorname{order}\left(f_{v_{L}}^{r e v}\right)=\operatorname{rev}\left(\operatorname{order}\left(f_{v_{L}}\right)\right)$. Similarly, $f_{v_{R}}^{r e v}=f_{v_{R}}$ and $\operatorname{order}\left(f_{v_{R}}^{r e v}\right)=\operatorname{rev}\left(\operatorname{order}\left(f_{v_{R}}\right)\right)$.

If $v$ is a product gate, then $f_{v}^{r e v}=f_{v_{R}}^{r e v} \times f_{v_{L}}^{r e v}$, which is equivalent to $f_{v_{R}} \times f_{v_{L}}=f_{v}$ by induction hypothesis. By induction hypothesis, $\operatorname{order}\left(f_{v}^{r e v}\right)$ is $\operatorname{order}\left(f_{v_{R}}^{r e v}\right)$ appended with $\operatorname{order}\left(f_{v_{L}}^{r e v}\right)$. The $\operatorname{order}\left(f_{v_{L}}^{r e v}\right)=\operatorname{rev}\left(\operatorname{order}\left(f_{v_{L}}\right)\right)$, and $\operatorname{order}\left(f_{v_{R}}^{r e v}\right)=\operatorname{rev}\left(\operatorname{order}\left(f_{v_{R}}\right)\right)$. Therefore, $\operatorname{order}\left(f_{v}^{r e v}\right)=\operatorname{rev}\left(\operatorname{order}\left(f_{v}\right)\right)$.

If $v$ is a sum gate, then $f_{v}^{r e v}=f_{v_{L}}^{r e v}+f_{v_{R}}^{r e v}$, which is equivalent to $f_{v_{L}}+f_{v_{R}}=f_{v}$ by induction hypothesis. As $v$ is a sum gate, $\operatorname{order}\left(f_{v}\right)=$ $\operatorname{order}\left(f_{v_{L}}\right)=\operatorname{order}\left(f_{v_{R}}\right)$. As order $\left(f_{v_{L}}^{r e v}\right)=\operatorname{rev}\left(\operatorname{order}\left(f_{v_{L}}\right)\right)$ by induction hypothesis, we have that $\operatorname{order}\left(f_{v}^{r e v}\right)=\operatorname{rev}\left(\operatorname{order}\left(f_{v}\right)\right)$ and $\operatorname{order}\left(f_{v_{R}}^{r e v}\right)=$ $\operatorname{rev}\left(\operatorname{order}\left(f_{v_{R}}\right)\right)$. Thus, order $\left(f_{v}^{r e v}\right)=\operatorname{order}\left(f_{v_{L}}^{r e v}\right)=\operatorname{order}\left(f_{v_{R}}^{r e v}\right)$.

The size of $C^{r e v}$ is same as that of $C$ because the only modification we are doing to $C$ is swapping the children of product gates. This completes proof of the lemma.

Using Lemma 1, we can handle the monotonically decreasing sequence without modifying the polynomial computed by a regular set-multilinear circuit. This gives us a circuit $\widehat{C}$ that computes the permanent polynomial of degree $\frac{\sqrt[4]{n}}{2}$. We remark that Lemma 1 can be adapted for non-commutative 
circuits as well.

We now explain how to get the permanent polynomial of degree $\frac{\sqrt{n}}{2}$ instead of $\frac{\sqrt[4]{n}}{2}$. This gives us quadratic improvement in the degree of the permanent polynomial. This is based on the observation that if $C$ is a regular set-multilinear circuit w.r.t a permutation $\sigma \in S_{n}$ computing the determinant polynomial $D E T_{n}(X)$, then for any permutation $\tau \in S_{n}$, there is another regular set-multilinear circuit $C^{\prime}$ w.r.t $\tau \circ \sigma \in S_{n}$ computing the same determinant polynomial $D E T_{n}(X)$. Moreover, the size of $C^{\prime}$ is at most one more than the size of $C$.

In other words, composition of permutations can be efficiently carried out for regular set-multilinear circuits computing the determinant polynomial $\operatorname{DET}_{n}(X)$.

Lemma 2 (Composition Lemma). Let $C=C_{1}+C_{2}$ be the sum of two regular set-multilinear circuits computing the determinant polynomial $D_{E} T_{n}(X)$, where the circuits $C_{1}, C_{2}$ are regular set-multilinear circuits w.r.t $\sigma_{1}, \sigma_{2} \in S_{n}$ respectively. Then for any permutation $\tau \in S_{n}$, there exists another circuit $C^{\prime}$ that computes $D E T_{n}(X) . C^{\prime}$ is also a sum of two regular set-multilinear circuits (regular set-multilinear w.r.t $\tau \circ \sigma 1, \tau \circ \sigma 2 \in S_{n}$ ). Moreover, the size of $C^{\prime}$ is at most one more than the size of $C$.

Proof. First, we describe the construction of the circuit $C^{\prime}=C_{1}^{\prime}+C_{2}^{\prime}$ and then prove its correctness.

Construction of $C^{\prime}$ : For every variable $x_{i, j}$ in $C=C_{1}+C_{2}$, substitute the variable $x_{\tau(i), j}$. Let $\widehat{C}$ be this modified circuit. If $\operatorname{sgn}(\tau)$ is -1 , then add a leaf node labeled -1 and multiply the root node of $\widehat{C}$ with this leaf node. Let $C^{\prime}$ be this modified circuit. The size of $C^{\prime}$ is at most one more than the size of $C$.

Correctness: Now we will prove that $C^{\prime}$ computes $D E T_{n}(X)$. Let $m_{1}$ and $m_{2}$ be any two monomials in $D_{E} T_{n}(X)$ computed by $C$. Let $m_{1}^{\prime}$ and $m_{2}^{\prime}$ be the monomials obtained by applying $\tau$ to the first index of each of the variables in $m_{1}$ and $m_{2}$ respectively. The permutations corresponding to $m_{1}^{\prime}$ and $m_{2}^{\prime}$ are $\tau \circ \sigma_{1}$ and $\tau \circ \sigma_{2}$ respectively.

- Case 1: $m_{1}=m_{2}$. We show that $m_{1}^{\prime}=m_{2}^{\prime}$ in $C^{\prime}$. We note that $m_{1}$ and $m_{2}$ could be computed by circuits $C_{1}$ and $C_{2}$ respectively. Thus, the order of variables appearing in $m_{1}$ and $m_{2}$ could be different in general. By construction of $C^{\prime}, x_{i, j}$ is substituted by the variable $x_{\tau(i), j}$. Since $m_{1}=m_{2}$, we get $m_{1}^{\prime}=m_{2}^{\prime}$.

- Case 2: $m_{1} \neq m_{2}$. We show that $m_{1}^{\prime} \neq m_{2}^{\prime}$ in $C^{\prime}$. Since $m_{1} \neq m_{2}$, there exists a variable $x_{i_{1}, j_{1}}$ in $m_{1}$ and a variable $x_{i_{2}, j_{2}}$ in $m_{2}$ such that 
$x_{i_{1}, j_{1}} \neq x_{i_{2}, j_{2}}$. Suppose $j_{1}=j_{2}$, then $i_{1} \neq i_{2}$. Then, $x_{\tau\left(i_{1}\right), j_{1}} \neq x_{\tau\left(i_{2}\right), j_{2}}$. This implies $m_{1}^{\prime} \neq m_{2}^{\prime}$. Suppose $j_{1} \neq j_{2}$, then $x_{\tau\left(i_{1}\right), j_{1}} \neq x_{\tau\left(i_{2}\right), j_{2}}$, which again implies that $m_{1}^{\prime} \neq m_{2}^{\prime}$.

By construction of $C^{\prime}$, we note that coefficients of monomials are not affected. Now we will prove that $C^{\prime}$ computes $D E T_{n}(X)$. Let $A_{X}$ be a $n \times n$ matrix where row $i$ contains all variables of the set $X_{i}$. In other words, the entry of $i$-th row and $j$-th column of the matrix $A_{X}$ is $x_{i, j}$. Let $\beta \in S_{n}$. By changing $x_{i, j}$ to $x_{\beta(i), j}$, in effect it permutes the rows of $A_{X}$. In other words, the determinant is equal to the determinant of $P_{\beta} \times A_{X}$, where $P_{\beta}$ is the $n \times n$ permutation matrix. The entry of $i$-th and $j$-th column of $P_{\beta}$ is 1 iff $j=\beta(i)$ and 0 otherwise. By Fact 1 and 2 , we have $\operatorname{det}\left(P_{\beta} \times A_{X}\right)=\operatorname{det}\left(P_{\beta}\right) \times \operatorname{det}\left(A_{X}\right)=\operatorname{sign}(\beta) \times \operatorname{det}\left(A_{X}\right)$.

Thus, composing the permutation $\tau$ with $\sigma_{1}, \sigma_{2}$ maps different monomials to different monomials and in effect does not change the determinant computed except that the sign changes. Note that $\operatorname{sgn}(\tau \circ \beta)=$ $\operatorname{sgn}(\tau) \cdot \operatorname{sgn}(\beta)$ (by Fact 3). Therefore, if $\operatorname{sgn}(\tau)=-1$, then the coefficients of $m_{1}^{\prime}$ and $m_{2}^{\prime}$ are the negatives of the coefficients of $m_{1}$ and $m_{2}$ respectively. Therefore, if $\operatorname{sgn}(\tau)=-1, C^{\prime}$ computes $D E T_{n}(X)$, as the leaf gate labeled -1 multiplied to the output gate ensures that $C^{\prime}$ computes $D E T_{n}(X)$. However, the coefficients of $m_{1}^{\prime}$ and $m_{2}^{\prime}$ are the same as the coefficients of $m_{1}$ and $m_{2}$ respectively, if $\operatorname{sgn}(\tau)=+1$. In the case that $\operatorname{sgn}(\tau)=+1$, there is no need of this leaf gate. In both cases, the polynomial computed by $C^{\prime}$ is $\operatorname{DET}_{n}(X)$.

Now we will show that $\operatorname{order}\left(C_{j}\right)=\left(\tau\left(\sigma_{j}(1)\right), \tau\left(\sigma_{j}(2)\right), \ldots, \tau\left(\sigma_{j}(n)\right)\right)$, $j \in\{1,2\}$. The proof is by induction on the depth $d$ of the circuit. We will prove it for $C_{1}$. The proof is similar for the circuit $C_{2}$. Recall that $C_{1}$ is regular set-multilinear circuit w.r.t $\sigma_{1}$. Let $v$ be a gate in the circuit. We denote polynomial computed at $v$ in $C$ and $C^{\prime}$ by $f_{v}$ and $f_{v}^{\prime}$ respectively.

Base Case: The base case is any node at depth 0, i.e, a leaf node. Let $\ell$ be any leaf node. Then $f_{\ell}$ is either a field constant or a variable $x_{i, j}$. If $f_{\ell} \in F$, then the $\operatorname{order}\left(f_{\ell}\right)$ is the empty sequence (). As there is no variable in $f_{\ell}$, there is no change to be made. Therefore, $\operatorname{order}\left(f_{\ell}^{\prime}\right)=()$, and therefore the claim trivially holds. If $f_{\ell}$ is a variable $x_{i, j}$, then $\operatorname{order}\left(f_{\ell}\right)=$ $(i)=\left(\sigma_{1}(k)\right)$, for some $k \in\{1,2, \ldots, n\}$. We change $x_{i, j}$ to $x_{\tau(i), j}$, which means $\operatorname{order}\left(f_{\ell}^{\prime}\right)=\left(\tau\left(\sigma_{1}(k)\right)\right)$.

Induction Hypothesis: Suppose the claim holds for any node at depth $d^{\prime}, 1 \leq d^{\prime}<d$.

Induction Step: Consider any node $v$ at depth $d^{\prime}+1$. Let $u$ and $w$ be its left and right children with degrees $d_{u}, d_{w}$ respectively. 
- Case 1: $v$ is a sum gate. Thus, $f_{v}^{\prime}=f_{u}^{\prime}+f_{w}^{\prime}$. Then $\operatorname{order}\left(f_{u}^{\prime}\right)=$ $\operatorname{order}\left(f_{w}^{\prime}\right)=\operatorname{order}\left(f_{v}^{\prime}\right)$.

- Case 2: $v$ is a product gate. Thus, $f_{v}^{\prime}=f_{u}^{\prime} \times f_{w}^{\prime}$. Let $0 \leq a \leq$ $n-d_{u}-d_{w}$, where $d_{u}, d_{w}$ denote degrees of $f_{u}, f_{w}$ respectively.

Let $\operatorname{order}\left(f_{u}\right)=\left(\sigma_{1}(a+1), \sigma_{1}(a+2), \cdots, \sigma_{1}\left(a+d_{u}\right)\right)$ and $\operatorname{order}\left(f_{w}\right)=\left(\sigma_{1}\left(a+d_{u}+1\right), \sigma_{1}\left(a+d_{u}+2\right), \cdots, \sigma_{1}\left(a+d_{u}+d_{v}\right)\right)$. By $\mathrm{IH}, \operatorname{order}\left(f_{u}^{\prime}\right)=\left(\tau\left(\sigma_{1}(a+1)\right), \tau\left(\sigma_{1}(a+2)\right), \ldots, \tau\left(\sigma_{1}\left(a+d_{u}\right)\right)\right)$, and let $\operatorname{order}\left(f_{w}^{\prime}\right)=\left(\tau\left(\sigma_{1}\left(a+d_{u}+1\right)\right), \tau\left(\sigma_{1}\left(a+d_{u}+2\right)\right), \ldots, \tau\left(\sigma_{1}\left(a+d_{u}+\right.\right.\right.$ $\left.\left.\left.d_{v}\right)\right)\right)$. Then $\operatorname{order}\left(f_{v}^{\prime}\right)=\left(\tau\left(\sigma_{1}(a+1)\right), \cdots, \tau\left(\sigma_{1}\left(a+d_{u}\right)\right), \tau\left(\sigma_{1}(a+\right.\right.$ $\left.\left.\left.d_{u}+1\right)\right), \cdots, \tau\left(\sigma_{1}\left(a+d_{u}+d_{v}\right)\right)\right)$.

Thus, in both cases, the claim holds. This completes the proof of the lemma.

Unlike Lemma 1, we note that in general this composition operation may not hold for any polynomial $f$ computed by a regular circuit. For example, if $C$ is a regular set-multilinear circuit computing the polynomial $f=x_{1,1} x_{2,0} x_{3,0} x_{4,1}$ then by swapping the $3 \mathrm{rd}$ and 4 th indices, we get a different polynomial $f^{\prime}=x_{1,1} x_{2,0} x_{4,0} x_{3,1}$. Now we have all results needed to the case where the determinant polynomial is computed by a sum of two regular set-multilinear circuits.

Theorem 3. Let $X=\left\{x_{i, j}\right\}_{i=1, j=1}^{n}$. If the determinant polynomial over $X$ is computed by a circuit $C$ of size $s$, where $C$ is the sum of two regular set-multilinear circuits, then the permanent polynomial of degree $\sqrt{n} / 2$ can be computed by a regular set-multilinear circuit $C^{\prime}$ of size polynomial in $n$ and $s$.

Proof. Let $C=C_{1}^{\sigma_{1}}+C_{2}^{\sigma_{2}}$, where the circuits $C_{1}^{\sigma_{1}}, C_{2}^{\sigma_{2}}$ are regular setmultilinear circuits w.r.t $\sigma_{1}, \sigma_{2} \in S_{n}$ respectively. We show that there is an efficient transformation that converts the given circuit $C$ to another circuit $C^{\prime}$ computing the permanent polynomial of degree $\sqrt{n} / 2$.

Without loss of generality, we can assume that $\sigma_{1}$ is the identity permutation. This is because otherwise by Lemma 2 we can get a new circuit $\hat{C}=C_{1}^{\sigma_{1}^{-1} \circ \sigma_{1}}+C_{2}^{\sigma_{1}^{-1} \circ \sigma_{2}}$ with $\sigma_{1}^{-1} \circ \sigma_{1}, \sigma_{1}^{-1} \circ \sigma_{2} \in S_{n}$ as the two permutations used. This does not increase the circuit size. By the Erdös-Szekeres Theorem, there is a monotone subsequence of length $\sqrt{n}$. Let $A$ be the set of all such indices.

- Case 1: Subsequence is increasing. As $\sigma_{1}$ is the identity, the same subsequence of indices in $\sigma_{1}$ is also increasing. We do the following 
substitutions. For all $j \notin A$, set $x_{j, j}=1$ and for all $i \in[n]$ and $i \neq j$, set $x_{j, i}=0$ and $x_{i, j}=0$. After this substitutions, the circuit computes the determinant polynomial over $A^{\prime}=\bigsqcup_{i \in A} X_{i}$ and the order of the subsequence in both $C_{1}$ and $C_{2}$ are the same. We rename the variable sets in $A^{\prime}$ as follows: if $i_{1} \in A_{1}$ is the $j$-th lowest index in the subsequence then we rename $X_{i_{1}}$ to $X_{j}$, and for all $k$, rename $X_{i_{1}, k}$ to $X_{j, k}$. The modified circuit $C^{\prime}$ computes the determinant polynomial over $\hat{X}=\bigsqcup_{i \in\left[n^{1 / 2}\right]} X_{i}$ and it is regular w.r.t the identity permutation in $S_{\sqrt{n}}$.

- Case 2: Subsequence is decreasing. Then by Lemma 1, we modify the circuit $C_{2}^{\sigma 2}$ to get a new circuit computing the same polynomial as computed by the circuit $C_{2}^{\sigma_{2}}$ but the new circuit is regular setmultilinear w.r.t the permutation $\sigma_{2}^{r e v}=\left(\sigma_{2}(n), \sigma_{2}(n-1), \cdots, \sigma_{2}(1)\right)$. We note that, by applying Lemma 1, no sign change occurs to the determinant polynomial. In this modified (second) circuit, the corresponding subsequence now becomes increasing. This reduces this case to case 1 .

Thus, after this modifications we have a new regular circuit $C^{\prime}$, that computes the determinant polynomial of degree $\sqrt{n}$, w.r.t the identity permutation. If $\lfloor\sqrt{n}\rfloor$ is not an even number then we substitute variables in $X_{\sqrt{n}}$ as explained before. Thus, $C^{\prime}$ computes the determinant polynomial of even degree. Now by the result of [2], we can compute the permanent polynomial of degree $\frac{\sqrt{n}}{2}$ by a circuit of size polynomial in $s$ and $n$. This completes the proof of the theorem.

\section{Hardness of the Determinant: Sum of Constantly-Many Regular Set-Multilinear cir- cuits}

In this section, we show that if the determinant polynomial $\operatorname{DET}_{n}(X)$ is computed by a sum of constantly many regular set-multilinear circuits then the permanent polynomial $P E R M_{n^{\epsilon} / 2}(X), \epsilon>0$ depends on $k$, computed a regular circuit. The proof of the following lemma is omitted due to lack of space. This is a generalization of the (composition) Lemma 2 but idea of the proof is similar.

Lemma 3. Let $C=C_{1}+C_{2}+\cdots+C_{k}$ be a sum of $k$ regular set-multilinear circuits such that $C$ computes $D E T_{n}(X)$. Let $C_{1}, C_{2}, \ldots, C_{k}$ be regular set- 
multilinear w.r.t $\sigma_{1}, \sigma_{2}, \ldots, \sigma_{k}$ respectively, where each $\sigma_{i} \in S_{n}$. For any $\tau \in S_{n}$, let $C_{1}^{\tau\left(\sigma_{1}\right)}, C_{2}^{\tau\left(\sigma_{2}\right)}, \ldots ., C_{k}^{\tau\left(\sigma_{k}\right)}$ be the circuits obtained by substituting $x_{\tau(i), j}$ for each variable in $x_{i, j}$ in each of the $k$ circuits. Let $C^{\prime}$ be the sum of $C_{1}^{\tau\left(\sigma_{1}\right)}, C_{2}^{\tau\left(\sigma_{2}\right)}, \ldots, C_{k}^{\tau\left(\sigma_{k}\right)}$. Then $C^{\prime}$ also computes $\operatorname{DET}_{n}(X)$. Moreover, the size of $C^{\prime}$ is at most one more than the size of $C$.

Without loss of generality we can assume that for each $i \neq j \in[k]$, $\sigma_{i} \neq \sigma_{j}$. Otherwise, we can combine all $C_{i}$ 's which use same $\sigma_{i}$ into a single $C_{i}$ using addition gates and get a circuit $C$ that is a sum of $k^{\prime}$ regular setmultilinear circuits, where $k^{\prime}<k$. Therefore, $C$ is the sum of $k^{\prime}$ regular set-multilinear circuits such that no two permutations used by any two of these $k^{\prime}$ circuits is same. We call such a circuit $C$ as $k^{\prime}$-regular circuit.

Theorem 4. Let $C$ be the sum of k-many regular set-multilinear circuits, of size s, computing the determinant polynomial $D E T_{n}(X)$. Then there exists a regular set-multilinear circuit whose size is at most $s+1$ that computes the determinant polynomial $D E T_{n^{\epsilon}}\left(X^{\prime}\right)$, where $X^{\prime}=\left\{x_{i, j}\right\}_{i=1, j=1}^{n^{\epsilon}}$ and $\epsilon \geq$ $1 / 2^{k-1}$.

Proof. Let $C=C_{1}^{\sigma_{1}}+C_{2}^{\sigma_{2}}+\cdots+C_{k}^{\sigma_{k}}$, where the circuits $C_{i}^{\sigma_{i}}$ are regular set-multilinear circuits w.r.t $\sigma_{i} \in S_{n}, i \in[k]$. We show that there is an efficient transformation that converts the given circuit $C$ to another circuit $C^{\prime}$ computing the determinant polynomial of degree $n^{\epsilon}, \epsilon=1 / 2^{k-1}$.

Without loss of generality, we can assume that $\sigma_{1}$ is the identity permutation. This is because otherwise by Lemma 2 we can get a new circuit $\hat{C}=\hat{C}_{1}+\hat{C}_{2}+\cdots+\hat{C}_{k^{\prime}}$ where $\hat{C}_{i}$ is a regular set-multilinear circuit w.r.t the permutation $\sigma_{1}^{-1} \circ \sigma_{i} \in S_{n}$, where $i \in[k]$. We note that $\hat{C}$ computes the same polynomial as circuit $C$ and both circuits have the same size.

Denote by $C^{(\ell)}$ the circuit obtained after the $\ell$-th iteration, where $0 \leq$ $\ell<k$. We will show that $C^{(\ell)}$ computes the determinant polynomial of degree $n^{1 / 2^{\ell}}$ and $C^{(\ell)}$ is a $(k-\ell)$-regular circuit.

At iteration 0 , this condition holds, as $C^{(0)}=C$ computes the determinant polynomial over $X$ and $C^{(0)}$ is a $k$-regular circuit.

Suppose the condition is true for some $m$, where $0 \leq m<k$. We will show that $C^{(m+1)}$ computes the determinant polynomial of degree $n^{1 / 2^{m+1}}$ and $C^{(m+1)}$ is a $k-(m+1)$-regular circuit. Note that $C_{1}, C_{2}, \cdots, C_{k}$ have been modified during the first $m$ iterations. Let us denote these modified circuits at the end of the $m$-th iteration by $C_{1}^{\prime}, C_{2}^{\prime}, \cdots, C_{k}^{\prime}$. Thus, $C^{(m)}=$ $C_{1}^{\prime}+C_{2}^{\prime}+\cdots+C_{k}^{\prime}$.

Without loss of generality, we will assume that each variable in the determinant computed by $C^{(m)}$ has both its indices in $X^{(m)}=\left\{1,2, \cdots, k_{m}\right\}$, 
where $k_{m}=n^{\frac{1}{2^{m}}}$. We note that the first $m$ regular set-multilinear circuits $C_{1}^{\prime}, C_{2}^{\prime}, \cdots, C_{m}^{\prime}$ are regular w.r.t identity permutation $i d \in S_{k_{m}}$. As noted before, we can combine all $C_{i}^{\prime}$ 's which has same $\sigma_{i}$ as single $C_{i}$ using addition gates. By Erdös-Szekeres Theorem [4], in $\sigma_{m+1}^{\prime}$, there is a monotone subsequence of length $n^{\frac{1}{2^{m+1}}}$. There are two cases to handle based on whether the subsequence is increasing or decreasing.

- Case 1: Suppose $\sigma_{m+1}^{\prime}$ has an increasing subsequence. Let $S^{(m+1)}=$ $\left\{i_{1}, i_{2}, \cdots, i_{k_{m+1}}\right\}$ be the set of indices in this increasing subsequence, where $k_{m+1}=n^{\frac{1}{2^{m+1}}}$. We do the following substitutions. For all $j \notin S^{(m+1)}$, set $x_{j, j}=1$ and for all $i \in\left[k_{m}\right]$ and $i \neq j$, set $x_{j, i}=0$ and $x_{i, j}=0$. After these substitutions, the circuit computes the determinant polynomial over $A^{\prime}=\bigsqcup_{i \in S^{(m+1)}} X_{i}$. We rename the variable sets in $A^{\prime}$ as follows: if $i_{1} \in S^{(m+1)}$ is the $j$-th lowest index in the subsequence then we rename $X_{i_{1}}$ to $X_{j}$, and for all $k$, rename $x_{i_{1}, k}$ to $x_{j, k}$. The modified circuit $C^{(m+1)}$ computes the determinant polynomial over $\hat{X}=\bigsqcup_{i \in\left[k_{m+1}\right]} X_{i}$. It is clear that $\sigma_{1}^{\prime}=\sigma_{2}^{\prime}=\cdots=\sigma_{m}^{\prime}=\sigma_{m+1}^{\prime}=i d e n t i t y$. This shows that $C^{(m+1)}$ is a $k-(m+1)$-regular circuit.

- Case 2: Suppose $\sigma_{m+1}^{\prime}$ has only a decreasing subsequence, then, we modify the sub-circuit $C_{m+1}^{\prime}$ by Lemma 1 to get a new circuit computing the same polynomial as computed by the $(m+1)$-th subcircuit in the previous iteration but the new circuit is regular setmultilinear w.r.t the permutation $\sigma_{m+1}^{r e v}=\left(\sigma_{m+1}^{\prime}\left(k_{m}\right), \sigma_{m+1}^{\prime}\left(k_{m}-\right.\right.$ $\left.1), \cdots, \sigma_{m+1}^{\prime}(1)\right)$. Note that after reversal operation, Lemma 1 guarantees that the polynomial computed by the circuit $C_{m+1}^{\prime}$ does not change. In $\sigma_{m+1}^{r e v}$, the corresponding subsequence now becomes increasing. It is clear that the same sequence of indices in $\sigma_{1}^{\prime}, \sigma_{2}^{\prime}, \cdots, \sigma_{m}^{\prime}$ are also increasing. This reduces this case to case 1.

Clearly, $C^{(m+1)}$, obtained at the end of the $(m+1)$-th iteration, computes the determinant over $X^{(m+1)}=\left\{x_{i, j} \mid i, j \in S^{(m+1)}\right\}$. This implies that at the end of $(k-1)$-th iteration, $C^{(k-1)}$ computes the determinant of degree $n^{\epsilon}$ over $X^{(k-1)}$, where $\epsilon=1 / 2^{k-1}$. Moreover, $C^{(k-1)}$ is a 1 -regular setmultilinear circuit. This completes the proof of the theorem.

Let $d$ be the degree of the determinant polynomial computed by the circuit $C^{(k-1)}$ in the above theorem. Clearly, $d \geq n^{\frac{1}{2^{k-1}}}$. If $\lfloor d\rfloor$ is not an even number then like before we substitute variables in the set $X_{\lfloor d\rfloor}$ such that 
the modified circuit computes the determinant of even degree $\lfloor d\rfloor-1$. Now by the result of [2], we can compute the permanent polynomial of degree $d / 2$ by a circuit of size polynomial in $s$ and $n$. Thus, we get the following main result as a corollary.

Corollary 1. Let $C$ be the sum of k-many regular set-multilinear circuits computing the determinant polynomial $D E T_{n}(X)$. Let $s$ denote the size of the circuit $C$. Then there exists a regular set-multilinear circuit $\widehat{C}$ computing the permanent polynomial PER $M_{n^{\epsilon} / 2}$, where $\epsilon=1 / 2^{k-1}$. Moreover, the size of $\hat{C}$ is polynomial in $s$ and $n$.

We note that to compute the permanent polynomial of degree $n$, we need to consider the determinant polynomial of degree $n^{2^{k-1}}$ computed by a $k$-regular circuit. So, our methods need $k$ to be a constant.

\section{Discussion}

In this paper we studied the complexity of computing the determinant polynomial using sum of constant number of regular set-multilinear circuits. We showed that computing the determinant in this model is at least as hard as computing the commutative permanent polynomial. An interesting open question is whether our results can be extended to the sum of a non-constant (some function of the degree of the determinant) number of regular set-multilinear circuits. Another question is: What is the complexity of computing the determinant polynomial using set-multilinear circuits?. This question was also raised in [1].

\section{References}

[1] Arvind, V., Raja, S.: Some lower bound results for set-multilinear arithmetic computations. Chicago Journal of Theoretical Computer Science 2016(6)

[2] Arvind, V., Srinivasan, S.: On the hardness of the noncommutative determinant. In: Proceedings of the 42nd ACM Symposium on Theory of Computing, STOC 2010, Cambridge, Massachusetts, USA, 5-8 June 2010. pp. 677-686 (2010)

[3] Berkowitz, S.J.: On computing the determinant in small parallel time using a small number of processors. Inf. Process. Lett. 18(3), 147-150 (1984) 
[4] Erdös, P., Szekeres, G.: A combinatorial problem in geometry. Compositio Mathematica 2, 463-470 (1935)

[5] Hrubes, P., Wigderson, A., Yehudayoff, A.: Non-commutative circuits and the sum-of-squares problem. In: Proceedings of the 42nd ACM Symposium on Theory of Computing, STOC 2010, Cambridge, Massachusetts, USA, 5-8 June 2010. pp. 667-676 (2010)

[6] Nisan, N.: Lower bounds for non-commutative computation (extended abstract). In: STOC. pp. 410-418 (1991)

[7] Nisan, N., Wigderson, A.: Lower bounds for arithmetic circuits via partial derivatives (preliminary version). In: 36th Annual Symposium on Foundations of Computer Science, Milwaukee, Wisconsin, 23-25 October 1995. pp. 16-25 (1995)

[8] Raz, R.: Multi-linear formulas for permanent and determinant are of super-polynomial size. J. ACM 56(2) (2009)

[9] Valiant, L.G.: Completeness classes in algebra. In: Proceedings of the 11h Annual ACM Symposium on Theory of Computing, April 30 - May 2, 1979, Atlanta, Georgia, USA. pp. 249-261 (1979)

[10] Valiant, L.G.: The complexity of computing the permanent. Theor. Comput. Sci. 8, 189-201 (1979) 